\title{
ADAPTACIÓN Y VALIDACIÓN DEL CUESTIONARIO DE AUTOEVALUACIÓN PRENATAL EN GESTANTES USUARIAS DEL INSTITUTO NACIONAL MATERNO PERINATAL
}

\author{
Juan José Gabriel Artica Martinez ${ }^{1}$
}

\begin{abstract}
RESUMEN
El presente trabajo de investigación, de tipo instrumental, tiene por objetivo adaptar y validar el Cuestionario de autoevaluación prenatal (PSQ) en una muestra de 790 gestantes usuarias del Instituto Nacional Materno Perinatal, con edades comprendidas entre 13 y 45 años $(M=23.8, D E=6.7)$. Se desarrollaron evidencias de validez respecto al contenido y a la estructura interna del cuestionario; en cuanto a lo primero, se utilizó la $\vee$ de Aiken y los intervalos de confianza, encontrando 69 ítems del cuestionario con IC superiores a .75, lo cual indica acuerdo de los jueces expertos sobre la relevancia de los ítems. Respecto al análisis de la estructura interna, se realizó el análisis factorial exploratorio encontrando un $\mathrm{KMO}=.97 ; \mathrm{X}^{2}(45)=$ $49430.3, p<.001$; posteriormente se comparó, a través de un análisis factorial confirmatorio, el modelo teórico propuesto por la autora con el modelo resultante del AFE el cual sugirió cinco dimensiones. Se observó un mejor ajuste en el modelo de cinco dimensiones en su versión de 30 ítems $x 2=787.75, x 2 / g l=1.99, \mathrm{CFI}=.979$, RMSEA $=.035$ [.032- .039], SRMR $=.038, \mathrm{AlC}=927.75$. Se estimó los coeficientes de confiabilidad para cada una de las cinco dimensiones encontrándose valores mayores a .92 los cuales son considerados aceptables. Se concluye que el instrumento resultante, con base en un modelo multidimensional de cinco factores, presenta adecuadas propiedades psicométricas en cuanto a su validez y confiabilidad, por lo que su uso es aceptable como un instrumento para conocer aspectos psicosociales relacionados a la adaptación al embarazo.
\end{abstract}

Palabras claves: gestante, autoevaluación, psicosocial, validez, confiabilidad (Fuente: DeCS BIREME).

\section{ADAPTATION AND VALIDATION OF THE SELF-ASSESSMENT QUESTIONNAIRE PRENATAL IN USER MANAGERS OF THE NATIONAL INSTITUTE PERINATAL MATERNAL}

\begin{abstract}
The objective of this research work, of an instrumental nature, is to adapt and validate the Prenatal Self-Assessment Questionnaire (PSQ) in a sample of 790 pregnant women using the National Perinatal Maternal Institute, aged between 13 and 45 years $(M=23.8, D E=6.7)$. Evidence of validity will be developed regarding the content and internal structure of the questionnaire; As for the first, the $\mathrm{V}$ of Aiken and the confidence intervals are detailed, 69 items of the questionnaire with $\mathrm{Cl}$ greater than .75 have been found, and it has been indicated with respect to the importance of the items. Regarding the analysis of the internal structure, exploratory factor analysis was performed finding a $\mathrm{KMO}=.97 ; \mathrm{X}^{2}(45)=49430.3, p<.001$; Subsequently, the theoretical model provoked by the author with the resulting model of the AFE, which has five dimensions, was compared through a confirmatory factor analysis. A better configuration is seen in the five-dimensional model in its version of 30 items $X 2=787.75, X 2 / \mathrm{gl}=1.99, \mathrm{CFI}=.979$, RMSEA $=.035[.032-039]$, SRMR $=.038, \mathrm{AIC}=927.75$. Reliability coefficients were estimated for each of the five dimensions. It is concluded that the resulting instrument, based on a multidimensional model of five factors, presents the psychometric properties in terms of its validity and reliability, why its use is acceptable as an instrument to know psychosocial aspects related to the adaptation to pregnancy.
\end{abstract}

Key words: pregnant, self-assessment, psychosocial, validity, reliability (Source: MeSH NLM).

\section{INTRODUCCIÓN}

La maternidad, es un proceso largo cuyo origen se inicia mucho antes de la llegada específica del parto. "El núcleo psicológico de la maternidad no empieza en el momento del embarazo, sino que comienza a organizarse en la infancia a través de la relación con la propia madre y a través de las fantasías de cómo ser madre a su vez"1. La maternidad en el transcurso de su evolución bilógica y en sus aspectos fisiológicos se desarrolla y se repite más o menos de la misma forma. Sin embargo, los aspectos psicológicos aquellos que convierten una maternidad distinta a otra $^{1,2,3}$. De ahí la necesidad de contar con instrumentos de medición, con evidencias de validez y confiabilidad para comprender este proceso tanto en sus aspectos psicológicos como sociales, es una labor necesaria. Por lo mencionado, podemos apreciar la necesidad de contar con un instrumento que nos permita explorar aspectos actitudinales, comportamentales y sociales en el proceso de adaptación al embarazo, encontrando el Cuestionario de autoevaluación prenatal (PSQ) diseñado por Regina

Citar como: Artica JJG. Adaptación y validación del cuestionario de autoevaluación prenatal en gestantes usuarias del instituto nacional materno perinatal. Rev Peru Investig Matern Perinat 2019; 8(4): 19-24 DOI https://doi.org/10.33421/inmp.2019169 
Lederman. ${ }^{4}$ De esta manera, reconociendo la importancia de la evaluación de la adaptación prenatal y siguiendo el objetivo propuesto por esta investigación formulamos la siguiente pregunta: ¿Cuáles son las evidencias de validez y confiabilidad de Cuestionario de autoevaluación del período prenatal de Lederman? Y para lo cual se planteó como objetivo principal: Adaptar y validar el Cuestionario de autoevaluación prenatal (PSQ) en gestantes adultas y adolescentes atendidas en el Instituto Nacional Materno Perinatal.

\section{MATERIALES Y MÉTODOS}

La presente investigación corresponde a un diseño de investigación instrumental 5,6 , dado que este trabajo analizan las propiedades psicométricas de instrumentos de medida psicológicos, ya sea de nuevos tests o de la traducción y adaptación de tests ya existentes. La población de estudio comprende a gestantes adultas y adolescentes que asisten a los consultorios gineco- obstétricos del Instituto Nacional Materno Perinatal, participaron en este estudio 790 gestantes $^{7}$. Se consideraron gestantes adultas y adolescentes (a partir de los 13 años), a partir del segundo trimestre de embarazo, que aceptaron participar de la investigación. Se excluyen a gestantes con alteraciones mentales, analfabetas y cuyo embarazo sea producto de abuso sexual.

La aplicación de la ficha sociodemográfica y el cuestionario de autoevaluación prenatal (PSQ) se realizó principalmente en las salas de espera de consultorios externos del área de gineco - obstetricia del Instituto Nacional Materno Perinatal. La aplicación se realizó individual y colectivamente. Igualmente, se solicitó autorización a la jefatura de obstetricia para ingresar a los ambientes de estimulación prenatal donde se logró aplicar los instrumentos de manera colectiva. Se aplicó un total de 790 cuestionarios entre gestantes adultas y adolescentes. Todas las participantes firmaron la ficha de consentimiento informado.

Para el análisis estadístico, se realizó en tres fases, en primer lugar, se realizó la adaptación lingüística del cuestionario revisando cada uno de los 79 ítems. En la segunda fase, se desarrollaron las evidencias de validez basadas en el contenido del cuestionario. Se utilizó la estrategia de criterio por jueces expertos a fin de evaluar la relevancia de cada ítem para el constructo al que pertenece. La tercera fase, se desarrollaron las evidencias de validez basadas en la estructura interna del instrumento.

En cuanto al análisis descriptivo de las características de las participantes se analizaron las opciones de media, desviación estándar, valores mínimos y máximos, frecuencias y porcentajes; asimismo, se exploró la estructura subyacente al cuestionario a fin de corroborar si coincide con la propuesta de la autora. Luego de someter los 79 ítems al análisis factorial exploratorio a través del programa FACTOR en su versión $10.8 .02{ }^{8}$ se obtuvo como sugerencia la existencia de seis factores. Con el propósito de contar con mayor evidencia respecto a la estructura interna, se estimó la capacidad discriminativa de cada uno de los ítems mediante la correlación ítemtest corregida, empleando el valor mínimo aceptado de $.20^{9}$, se obtuvo que 30 ítems evidencian poca capacidad discriminante y un menor aporte al valor total del instrumento.

En base a esta última información, se decidió realizar nuevamente un análisis factorial exploratorio con los 49 ítems resultantes.

En tercer lugar, se realizó el análisis factorial confirmatorio (AFC) a través del programa AMOS en su versión 24 , se utilizó el método de máxima verosimilitud para el cálculo de los diferentes índices de bondad de ajuste ${ }^{7,10-14}$ entre ellos X2, g.l., X2/g.I., CFI, RMSEA, RMR y AIC. Todos estos índices fueron necesarios para la estimación del mejor modelo.

Los resultados indicaron que el modelo de cinco dimensiones presenta mejores bondades de ajuste en comparación con los demás modelos. Durante el desarrollo de este análisis se eliminaron 19 ítems; por tanto, la estructura final retuvo a los cuatro factores agrupándose en ellos los 30 ítems. Se analizó la confiabilidad de las cinco dimensiones a través del índice omega ${ }^{15}$, el cual utilizó las cargas factoriales de los ítems.

En cuanto a la consideración ética, se solicitó a cada participante de la investigación el llenado de la Ficha de consentimiento informado, el cual señala los objetivos del estudio y la reserva de su identidad.

\section{RESULTADOS}

\section{Evidencias basadas en la validez de contenido}

El análisis de acuerdo entre los jueces se llevó a cabo a través de los valores obtenidos en la $\mathrm{V}$ de Aiken y sus Intervalos de confianza al $95 \%$ para cada uno de los ítems del cuestionario. Ocho ítems, según el proceso de validación de contenido del cuestionario, presentan un valor menor a .70 en el límite inferior del IC al 95\%. Por sugerencia de los autores consultados ${ }^{16}$ estos ítems tendrían que ser eliminados del cuestionario por poseer escasa representatividad a su dimensión; no obstante, serán incluidos en el análisis de validez de constructo del instrumento de medición, a través del análisis factorial exploratorio y confirmatorio, a fin de encontrar mayor evidencia de su poca contribución a cada una de las dimensiones a las que pertenecen.

\section{Evidencias de validez basadas en el constructo}

Para obtener evidencias de validez interna se inició con el análisis factorial exploratorio del instrumento, se utilizó el programa FACTOR en su versión 10.8.02 ${ }^{8}$. El análisis de los coeficientes de asimetría y curtosis ${ }^{11}$, para conocer la existencia de normalidad univariante, resultaron adecuados dentro del rango $+/-2^{17}$.

Igualmente, se analizó la multicolinealidad entre los ítems a fin de estimar la existencia de variables altamente correlacionadas o redundantes (correlaciones inter-ítem de $\geq 90)^{18}$.

Con el propósito de determinar la homogeneidad del instrumento se estimó la correlación ítem-test corregida. Los coeficientes obtenidos se ubican entre -.086 y .754. Siguiendo la propuesta de Kline, P. ${ }^{9}$ se encuentran 30 ítems cuyos valores de correlación respecto al cuestionario son menores de .20. Todos estos ítems evidencian poca capacidad discriminante y un menor aporte al valor total 
del instrumento.

Además, la prueba de esfericidad de Bartlett fue significativa $(x 2=49430.3 ; \quad g l=3081 ; \quad p<0,001) ; \quad$ los resultados obtenidos indican la posibilidad de realizar un análisis factorial. ${ }^{17}$
Se utilizaron matrices policóricas/tetracóricas en el análisis factorial debido a que los ítems están en un nivel de medición ordinal ${ }^{19}$

Tabla 1. Criterios para decidir del número de factores a extraer en el Cuestionario de autoevaluación prenatal- PSQ

\begin{tabular}{ccc}
\hline Criterio & $\begin{array}{c}\text { Numero de } \\
\text { factores }\end{array}$ & Comentario \\
\hline Kaiser & 11 & El décimo segundo valor es menor a 1. \\
Scree test & 6 & $\begin{array}{l}\text { La visualización de autovalores en la pendiente sugiere retenerse } 6 \text { factores, punto en } \\
\text { el cual la línea se torne recta. }\end{array}$ \\
Análisis paralelo & 6 & $\begin{array}{l}\text { Basado en la propuesta de Timmerman, M. E. \& Lorenzo- Seva, U. (2011) la estructura } \\
\text { subyacente al cuestionario sería de seis factores. } \\
\text { Criterio teórico }\end{array}$ \\
\hline
\end{tabular}

Con el propósito de contar con mayor evidencia para decidir el número de factores a extraer del cuestionario se decidió elaborar una matriz eliminando los 30 ítems con un valor menor a $.20^{9}$; es decir, una nueva matriz con 49 reactivos, se realizó el AFE con esta cantidad de ítems, igualmente se utilizaron matrices policóricas/tetracóricas ya que los ítems están en un nivel de medición ordinal; además del método de Implementación óptima de análisis paralelos y rotación Promin ${ }^{20}$.

El índice KMO mostró un valor de 0.97 y el test de Bartlett resultó estadísticamente significativo $(x 2=34415.7$; gl= 1176; $p$ < 0.001), lo que llevó a concluir que la aplicación del análisis factorial resultaba oportuno. Se decidió realizar un nuevo análisis factorial exploratorio contemplando 5 factores a fin de obtener una mejor distribución de los ítems. Para estimar las bondades de ajuste se revisaron seis indicadores, obteniéndose valores para los índices de ajuste absoluto como el estadístico ji cuadrado ( $x 2)$, la razón entre $x 2$ y el número de grados de libertad $(x 2)$ $\mathrm{gl})$, los índices SRMR y RMSEA. El índice de ajuste incremental $\mathrm{CFI}$; además se adjuntó el índice de ajuste de parsimonia de Aiken (AIC). El modelo compuesto por 49 ítems mostró mejores bondades de ajuste. Respecto a la razón entre $x 2$ y el número de grados de libertad $(x 2 / g l)$ se obtuvo un valor de 3.324, este valor debería ser menor a $2{ }^{18}$; sin embargo, los modelos en comparación ofrecen valores superiores al modelo de cinco factores.

Tabla 2. Índice de ajuste estadístico del Cuestionario de autoevaluación prenatal $(N=790)$ sin eliminación de ítems.

\begin{tabular}{lcccccc}
\hline Modelos & \multicolumn{1}{c}{ X2 } & X2/ gl & SRMR & CFI & AIC & RMSEA \\
\hline $\begin{array}{l}\text { Modelo original del PSQ- } \\
\text { relacionando dimensiones }\end{array}$ & 18765.9 & 6.295 & & 0.672 & 19123.9 & $.082[.081-.083]$ \\
\hline $\begin{array}{l}\text { Modelo original del PSQ- Bifactor } \\
\text { Modelo de 4 factores }\end{array}$ & 11418.0 & 3.906 & & 0.823 & 11892.006 & $.061[.060-.062]$ \\
\hline Modelo de 5 factores & 6065.3 & 5.411 & 0.0629 & 0.855 & 6273.264 & $.075[.073-.077]$ \\
\hline
\end{tabular}

Nota: $\mathrm{X} 2=$ Chi cuadrado, gl= grados de libertad, SRMR= raíz residual estandarizada cuadrática media, RMSEA= error cuadrático medio de aproximación, $\mathrm{CFI}=$ índice de ajuste comparativo, $\mathrm{AIC}=$ criterio de Akaiken, $\mathrm{IC}=$ intervalos de confianza.

Es importante resaltar que a pesar de que el modelo de cinco factores presentó mejores bondades de ajuste, dos de los indicadores analizados no cumplieron con los requerimientos del análisis factorial confirmatorio, la razón entre $x 2$ y el número de grados de libertad $(x 2 / g l<2)$ y el CFI (z.95). Por ello, se decidió iniciar el proceso de eliminación de ítems. El siguiente análisis se encuentra encaminado a obtener el modelo con mejores bondades de ajuste; para tal propósito se eliminó consecutivamente los ítems siguiendo de las sugerencias brindadas por el Índice de modificación del programa (AMOS), obteniendo en cada calculo mejores valores sobre los indicadores de ajuste. Este proceso llevó a la eliminación de 19 ítems, el modelo resultante conformado por 30 ítems ofrece los mejores valores.

Al realizar el análisis factorial confirmatorio (AFC) se comprobó el supuesto de normalidad multivariada mediante el coeficiente de Mardia, se obtuvo un valor de 45.35 el cual es inferior a $70{ }^{14}$. 
En cuanto a la razón entre $\mathrm{x} 2$ y el número de grados de libertad (x2/gl) se obtuvo un valor de 1.994, el cual se ubica por debajo de $2{ }^{18}$. Se obtuvo un SRMR $=.0383$ el cual ofrece un valor menor a $.08^{12}$. EI RMSEA exhibió un valor de 0.35 con un intervalo de confianza al 95\% (.032.039), al respecto se sugieren valores menores a $.06^{12}$.

Asimismo, se obtuvo un índice de ajuste incremental CFI= .979 el cual es mayor al valor sugerido. Por último, el índice de ajuste de parsimonia de Aiken se exhibe un valor inferior a los otros modelos analizados $(\mathrm{AIC}=927.753)$.

Como puede apreciarse el modelo final de 30 ítems cumple con todos los requerimientos de los indicadores más relevantes para este análisis factorial confirmatorio. Además, es importante mencionar que este modelo no contiene errores correlacionados en su estructura. Al respecto, el cuestionario resultante estaría compuesto por 5 factores los cuales comprenderían las siguientes dimensiones: las dimensiones Aceptación al embarazo se incluiría en la dimensión Identificación con el rol matero; igualmente la dimensión Miedo al dolor y a la pérdida de control durante el parto quedaría incluida en la dimensión Preparación para el parto. Las dimensiones restantes se mantendrían en la estructura final del instrumento: calidad de la relación con la madre, calidad de la relación con la pareja y preocupación por el bienestar propio y del bebé. Las dimensiones agrupadas presentan características orientadas a un mismo aspecto de medición.

La estructura resultante del cuestionario de autoevaluación prenatal mantiene la idea original de la autora del instrumento, reuniendo dimensiones que miden aspectos diferentes, pero interrelacionados, de la adaptación psicosocial del embarazo.

La presente tabla presenta los cinco factores que componen la estructura del modelo final cada una está conformada por 8, 6, 8, 4 y 4 ítems.

Tabla 3. Dimensiones del Cuestionario de autoevaluación prenatal compuesta por 30 ítems

\begin{tabular}{lc}
\hline F1. Identificación con el rol materno & $3,30,65,67,68,69,72,74$ \\
$\begin{array}{l}\text { F2. Preocupación por el bienestar propio y del bebé } \\
\text { F3. Preparación para el parto }\end{array}$ & $8,22,29,36,43,79$ \\
F4. Calidad de la relación con la madre & $6,18,19,39,46,54,57,76$ \\
F5. Calidad de la relación con la pareja & $14,21,28,49$ \\
\hline
\end{tabular}

Por último, se obtuvieron correlaciones positivas pequeñas de .198 y .291 entre el factor 2 y el factor 3 , y el factor 2 y el factor 5. Asimismo, se obtuvieron correlaciones positivas moderadas de .355 y .313 entre el factor 1 y el factor 2 , y el factor 2 y el factor 4 . Se apreciaron correlaciones positivas altas de .729; .769; .783; .571 y .617 entre el factor 1 y el factor 3 , el factor 1 y el factor 4 , el factor 1 y el factor 5 , el factor 3 y el factor 4 y el factor 3 y el factor 5 respectivamente.

En base a los hallazgos sobre la dimensionalidad el cuestionario, los cuales sugirien un modelo multidimensional, se calcularon los coeficientes de confiabilidad para las cinco dimensiones que la integran. El valor de la confiabilidad fue estimado a través del coeficiente omega ${ }^{15}$, el cual a diferencia del coeficiente alfa de Cronbach usa las cargas factoriales de los ítems(20); por lo tanto, los valores obtenidos resultan más estables y reflejan un mejor nivel de fiabilidad 20 . Asimismo, es importante resaltar que el cálculo de este coeficiente no depende del número de ítems ${ }^{15}$.

En cuanto a los valores obtenidos en cada una de las dimensiones del cuestionario, se encontraron valores entre 0.849 a .968, siendo las dimensiones Preparación para el parto e Identificación materna prenatal los coeficientes de menor y mayor valor respectivamente. Los coeficientes mostraron valores superiores a .70 por lo que fueron considerados aceptables.

Tabla 4. Confiabilidad de las puntuaciones en cada dimensión

\begin{tabular}{lcc}
\hline \multicolumn{1}{c}{ Dimensión } & Numero de ítems & Coeficiente omega \\
\hline F1. Identificación con el rol materno & 8 & 0.968 \\
\hline F2. Preocupación por el bienestar propio y del bebé & 6 & 0.85 \\
F3. Preparación para el parto & 8 & 0.849 \\
F4. Calidad de la relación con la madre & 4 & 0.937 \\
\hline F5. Calidad de la relación con la pareja & 4 & 0.926 \\
\hline
\end{tabular}


Se elaboraron los baremos de cada dimensión, construidos a partir de las puntuaciones brutas convertidas en percentiles $(\mathrm{Pc})$. Se construyó los baremos calculando los puntos de los percentiles $5,10,15, \ldots, 90,95$ y 99 como recomienda ${ }^{19}$

La dimensión Identificación con el rol materno obtuvo 32 como puntaje máximo y 8 como mínimo $(M=22.39$; $D E=9.62)$. La dimensión, Preocupación por el bienestar propio y del bebé obtuvo 24 como puntaje máximo y 7 como mínimo $(\mathrm{M}=13.88 ; \mathrm{DE}=4.62)$. La dimensión Preparación para el parto, se obtuvo 32 como puntaje máximo y 10 como mínimo $(M=20.70 ; D E=5.92)$. La dimensión Calidad de la relación con la madre obtuvo 16 como puntaje máximo y 4 como mínimo $(\mathrm{M}=10.68$; $\mathrm{DE}=4.78$ ), y por último, en la dimensión Calidad de la relación con la pareja se obtuvo 16 como puntaje máximo y 4 como mínimo $(M=10.58 ; D E=4.61)$.

Finalmente, como recomienda ${ }^{19}$ se consideraron como referencia el Pc 25 y el Pc 75 para definir los puntos de corte de los niveles bajo y alto respectivamente.

\section{DISCUSIÓN}

Respecto a las evidencias sobre la validez del constructo del cuestionario, inicialmente el instrumento propone una estructura de 7 dimensiones o sub escalas ${ }^{4}$, luego del estudio de Armegol, et al. (2007) esta se redujo a 6 dimensiones, siendo la sub escala Miedo al dolor y a la pérdida de control durante el parto incluida en la dimensión Preparación para el parto, suceso que coincide en nuestra investigación. Igualmente, los resultados del AFC concluyen la inclusión de los ítems de la dimensión Aceptación al embrazo en la dimensión Identificación con el rol materno.

Se unificaron dimensiones que mantienen una relación estrecha entre ellas, la dimensión Aceptación al embarazo con la dimensión Identificación con el rol materno; y la dimensión Miedo al dolor y a la pérdida de control durante el parto con la dimensión Preparación para el parto, al parecer para las gestantes, de nuestro contexto, muchos de los ítems de estas dimensiones se relacionan de tal forma que no miden aspectos diferentes. Por tanto, el instrumento resultante denominó a estas dimensiones como Identificación con el rol materno y Preparación para el parto.

Las evidencias de nuestro estudio parecen contribuir a la propuesta de Armegol, et al. (2007) quienes señalan que las dimensiones planteadas por Lederman representan indicadores de un constructo que las contiene; es decir, plantear la unidimensionalidad del constructo. A este argumento señalaremos que durante el proceso de validación se eliminaron 49 ítems del cuestionario original y dos dimensiones fueron incluidas en otras; asimismo, los aportes de los índices estadísticos del modelo bifactor no favorecieron a este modelo. Por tanto, a modo de contribuir con la línea de investigación en la validación de este instrumento, podría desarrollarse estudios en nuestra población, que aporten mayor evidencia contundente respeto al tema. De momento, bastará con asumir el modelo de cinco dimensiones resultante de este estudio.

\section{CONCLUSIONES}

El Cuestionario de autoevaluación prenatal (PSQ) ajusta adecuadamente a un modelo de cinco dimensiones con índices de confiabilidad aceptables; por tanto, puede considerarse como un instrumento con adecuadas propiedades psicométricas.

La evidencia basada en el contenido sugiere la eliminación de diez de los reactivos del cuestionario original, a pesar de la reformulación en la redacción de los ítems y el doble proceso de calificación al que fueron sometidos los ítems. El criterio para esta decisión se basa un valor menor a .70 en el límite inferior del IC al 95\% de estos ítems, lo cual mostraría la escasa representatividad de estos a su dimensión (Penfield \& Giacobbi, 2004).

En cuanto a su estructura interna, el análisis factorial exploratorio sugirió un número de factores diferente a la propuesta teórica del cuestionario. Se encontró una estructura de cinco factores la cual agrupó parsimoniosamente los ítems según la dimensión de medición. Las dimensiones Aceptación al embarazo e Identificación con el rol materno terminaron unificándose; al igual que las dimensiones Preparación para el parto y Miedo y pérdida de control. Por otro lado, el análisis factorial confirmatorio, a través del método de ecuaciones estructurales, ratificó la existencia de cinco dimensiones interrelaciones. El modelo final compuesto por 30 ítems, con alta capacidad discriminativa, cumplió con todos los índices de ajuste propios de este tipo de análisis.

La eliminación de los ítems de la versión original del Cuestionario de autoevaluación prenatal se debió principalmente a la redundancia en el contenido de los ítems y a la falta de adecuación al contexto de habla hispana ${ }^{21}$. Asimismo, la evidencia obtenida empíricamente no permitió sostener la propuesta jerárquica del modelo; por lo tanto, una interpretación de un modelo multidimensional impresiona ser la más pertinente. La dimensión Aceptación al embarazo mostró dificultades en el ajuste a una estructura jerárquica; por lo que se sugiere la necesidad de redefinir los ítems que integran esta dimensión.

Los hallazgos apoyaron parcialmente la versión original de Lederman quien planteó la existencia de siete dimensiones en la adaptación psicosocial al embarazo, la investigación solo validó cinco de ellas. Presentando un modelo quintudimensional de la adaptación psicosocial al embarazo. Se propone nominar a las dimensiones integradas como Identificación materna prenatal y Disposición positiva hacia al parto.

Se elaboraron baremos en base a percentiles para cada una de las dimensiones que conforman el cuestionario. Además, se propusieron puntos de corte Pc 25 y el Pc 75 para ubicar un nivel bajo y alto en la medición de los cinco aspectos psicosociales en la adaptación al embarazo.

\section{CONTRIBUCIÓN DEL ESTUDIO EN LA SALUD MATERNO PERINATAL}

Este estudio representa un punto de partida en el desarrollo de una línea de investigación en salud materna en el Instituto Nacional Materno Perinatal con énfasis en los aspectos psicológicos implicados en el proceso de maternización; contar con un instrumento adaptado y 
con evidencias de validez en población peruana podría facilitar el desarrollo de futuros estudios de carácter multidisciplinarios e interdisciplinarios.

Financiamiento: Autofinanciado

Conflicto de interés: El autor declara no tener algún conflicto de interés.

\section{REFERENCIAS BIBLIOGRÁFICAS}

1. María Pierola Galvez. Zelaya, C. R., Mendoza, J. \& Soto, E.(2006). La maternidad y sus vicisitudes hoy. Lima: SIDEA 291 p. Rev Psicol [Internet]. 27 de marzo de 2006 [citado 26 de noviembre de 2019];24(2). Disponible en: http://revistas. pucp.edu.pe/index.php/psicologia/article/view/1456

2. Jurado Garcia E. Los defectos al nacer. Un problema de salud publica en México. Panorama de la salud perinatal en México. Gac Médica México. 1996;132(2):141-51.

3. López MEG, Calva EA. Alteraciones psicológicas en la mujer con embarazo de alto riesgo. Psicol Salud. 2007;17(1):5361.

4. Lederman RP. Psychosocial adaptation in pregnancy: Assessment of seven dimensions of maternal development. Springer Publishing Co; 1996.

5. Manuel Ato, Juan J. López-García, Ana Benavente. A classification system for research designs in psychology. An Psicol Ann Psychol [Internet]. 20 de septiembre de 2013 [citado 26 de noviembre de 2019];29(3). Disponible en: https://revistas.um.es/analesps/article/view/ analesps.29.3.178511

6. Montero I, León OG. Clasificación y descripción de las metodologías de investigación en Psicología. Int J Clin Health Psychol. 2002;2(3):503-8.

7. Lloret-Segura S, Ferreres-Traver A, Hernández-Baeza A, Tomás-Marco I. El análisis factorial exploratorio de los ítems: una guía práctica, revisada y actualizada. An Psicol Psychol. 2014;30(3):1151-69.

8. Lorenzo-Seva U, Ferrando PJ. FACTOR: A computer program to fit thet exploratory factor analysis model. Behav Res Methods. 2006;38(1):88-91.

9. Kline P. A handbook of test construction (psychology revivals): introduction to psychometric design. Routledge; 2015.
10. Ferrando PJ, Anguiano-Carrasco C. EL ANÁLISIS FACTORIAL COMO TÉCNICA DE INVESTIGACIÓN EN PSICOLOGÍA. Papeles Psicólogo. 2010;31(1):18-33.

11. Hair JF, Anderson RE, Tatham RL, Black WC. Análisis multivariante. Vol. 491. Prentice Hall Madrid; 1999.

12. $\mathrm{Hu} \mathrm{L}$, Bentler PM. Fit indices in covariance structure modeling: Sensitivity to underparameterized model misspecification. Psychol Methods. 1998;3(4):424.

13. Manzano A, Zamora S, Salvador R. Sistema de ecuaciones estructurales: una herramienta de investigación. México DF: Centro Nacional de Evaluación para la Educación Superior; 2010.

14. Ruiz MA, Pardo A, San Martín R. Modelos de ecuaciones estructurales. Papeles Psicólogo. 2010;31(1):34-45.

15. McDonald RP. Test theory: A unified treatment. Psychology Press; 2013.

16. Penfield RD, Giacobbi PR. Applying a score confidence interval to Aiken's item content-relevance index. Meas Phys Educ Exerc Sci. 2004;8(4):213-25.

17. Muthén B, Kaplan D. A comparison of some methodologies for the factor analysis of non-normal Likert variables. $\mathrm{Br} \mathrm{J}$ Math Stat Psychol. 1985;38(2):171-89.

18. Tabachnick BG, Fidell LS, Ullman JB. Using multivariate statistics. Vol. 5. Pearson Boston, MA; 2007.

19. Lara SAD. ¿Matrices Policóricas/Tetracóricas o Matrices Pearson? Un estudio metodológico. Rev Argent Cienc Comport RACC. 2014;6(1):39-48.

20. Timmerman ME, Lorenzo-Seva U. Dimensionality assessment of ordered polytomous items with parallel analysis. Psychol Methods. 2011;16(2):209.

21. Armengol R, Chamarro A. Aspectos psicosociales de la gestación: El cuestionario de evalución prenatal. An Psicol Ann Psychol. junio de 2007;23(1):25-32.

\section{Correspondencia:}

Juan José Gabriel Artica Martinez

Dirección: Jr. Santa Rosa \# 941 - Cercado de Lima

Correo: juanartica@gmail.com

Teléfono: 940166793 